\title{
CAUSE-SPECIFIC MORTALITY OF OLDER ADULTS WITH AND WITHOUT DEPRESSIVE SYMPTOMS
}

\author{
P.D. St. John $n^{1,2}$, S.L. Tyas
}

\begin{abstract}
Objective: To determine which causes of death are most closely associated with depressive symptoms. Methods: 1751 community-living older adults were assessed in 1991 and followed five years later. Depressive symptoms were assessed with the Center for Epidemiologic Studies - Depression (CES-D) scale. Death certificates were reviewed independently by two reviewers. Multinomial logistic regression models were adjusted for age and gender, and constructed with specific causes of death as the outcome compared to the reference category of being alive at time 2. Results: Death certificates were available for 253 (59\%) of the 429 deceased participants. Those with depressive symptoms were more likely to die from cardiovascular diseases, stroke, respiratory diseases and non-specific syndromes, but not from infections or neoplasms. There were few deaths due to neurologic, renal, and gastrointestinal diseases. Conclusions: Depressive symptoms may be associated with death due to cardiovascular disease, stroke and respiratory disease.
\end{abstract}

Key words: Depression, depressive symptoms, CES-D, mortality, death, cause of death.

\section{Introduction}

Depression and depressive symptoms predict mortality in older adults (1). However, the causal pathway of this effect is unclear. Depressive symptoms may predict death independently, or act indirectly by altering health behaviours associated with mortality or by reflecting symptoms of illness in frail older adults who, in turn, have higher mortality. While depressive symptoms have been consistently shown to predict allcause mortality in older adults, whether they predict some causes of death more strongly than others is less clear. We therefore conducted an analysis of secondary data from the Manitoba Study of Health and Aging (MSHA) to determine which causes of death are associated with depressive symptoms.

\footnotetext{
1. Section of Geriatric Medicine, Department of Medicine, University of Manitoba,
Canada; 2. Centre on Aging, University of Manitoba, Canada; 3. School of Public

1. Section of Geriatric Medicine, Department of Medicine, University of Manitoba,
Canada; 2. Centre on Aging, University of Manitoba, Canada; 3. School of Public Health and Health Systems, University of Waterloo, Canada

Corresponding Author: Philip St. John, MD MPH FRCPC, Associate Professor, Head Section of Geriatrics, University of Manitoba, GE 547 Health Sciences Centre, 820 Sherbrook Street, Winnipeg, MB, Canada, R3A 1R9, Phone: (204) 787-3365, Fax: (204) 787-4826, Email: pstjohn@hsc.mb.ca
}

\section{Methods}

\section{Sample}

The MSHA is a cohort study of aging and cognition conducted in conjunction with the Canadian Study of Health and Aging (CSHA) (2). The MSHA began in 1991, when 1751 individuals aged 65 years and older living in the community were interviewed in person. These older persons were randomly selected according to health region and age group from a list provided by Manitoba Health. Since health care is universal in Manitoba, this is one of the most complete listings of residents available. The sampling frame was weighted by age groups to over-sample the older age groups. All health regions were represented, including rural areas. In 1996/1997, attempts were made to re-interview the 1751 individuals who had participated in 1991/1992. Death certificates were available for 253 (59\%) of the 429 individuals who had died by the end of the follow-up period. The cause of death was not recorded or was illegible on 6 certificates, and 170 of the participants did not give consent to have death certificate data collected. The flow of participants and the risk of mortality from depression are described elsewhere (4). For these analyses, we also included twelve participants who died after the second clinical follow-up, but for whom we have death certificate data (4). 


\section{Measures}

All participants were interviewed by trained interviewers in their homes using standardized measures. Age, gender, and education were self-reported. Depressive symptoms were measured using the Center for Epidemiologic Studies - Depression (CES-D) scale(3), with scores greater than 15 indicating the presence of depressive symptoms.

\section{Cause of Death}

The immediate cause of death, secondary cause of death, ancillary causes of death, and other factors leading to death were all coded using ICD-9CM codes. Coding was done independently by two of the study investigators, who then met together to achieve consensus. A third investigator was to review cases which could not be resolved, but there were no situations where this was necessary. The reviewers did not have access to screening questionnaire or clinical data when reviewing the death certificates. The reviewers used ICD 9CM codes. For these analyses, cause of death was categorized as missing, infectious diseases (which included pneumonia), neoplasms, neurologic diseases (which included dementia, seizures and multiple sclerosis but excluded stroke), cardiovascular disease (which included ruptured abdominal aneurysms as well as acute myocardial infarction, heart failure and ischemic heart disease), stroke, respiratory conditions (which included chronic obstructive pulmonary disease and respiratory failure), gastrointestinal diseases (which included gastrointestinal bleeding, cirrhosis, colitis and pancreatitis), renal failure, and non-specific conditions (which included dehydration and ICD-9CM codes 780-799).

\section{Analysis}

Chi-square tests and analysis of variance (ANOVA) were used for categorical variables, and Student's t-tests (assuming unequal variance) were used for continuous variables. Multinomial logistic regression models were adjusted for age and gender, and constructed with specific causes of death as the outcome compared to the reference category of being alive at time 2 . The category "missing" was also considered as an outcome.

\section{Results}

The mean age of the 1751 participants was 76.2 years (standard deviation (SD) 7.1); $58.3 \%$ were women. The mean educational attainment was 9.3 (SD 3.6) years. At time 1, there were 1509 participants without depressive symptoms and 242 participants (13.8\%) with depressive symptoms. Those with depressive symptoms were more likely to have death certificate data missing.

Over the five-year interval, 429 participants died: $22.6 \%$ of those without depressive symptoms compared to $36.4 \%$ of those with depressive symptoms. The immediate causes of death are shown in the table. In

Table

Cause of death over the five year time period in community-living older adults with and without depressive symptoms

\begin{tabular}{|c|c|c|c|c|}
\hline $\begin{array}{l}\text { Cause of Death (ICD- } \\
9 \mathrm{CM})\end{array}$ & $\begin{array}{l}\text { No Depressive Symp- } \\
\text { toms }(\mathrm{N}=1509)\end{array}$ & $\begin{array}{l}\text { Depressive Symptoms } \\
\qquad(\mathrm{N}=242)\end{array}$ & Total Sample $(\mathrm{N}=1751)$ & $\begin{array}{c}\text { Risk of death (refe- } \\
\text { rence = alive) OR }(95 \% \\
\text { CI) }\end{array}$ \\
\hline Alive at time 2 & $1168(77.4 \%)$ & $154(63.4 \%)$ & $1322(75.5 \%)$ & \\
\hline Dead at last contact & $341(22.6 \%)$ & $88(36.4 \%)$ & $429(24.5 \%)$ & \\
\hline Cause of death missing & $145(9.6 \%)$ & $31(12.8 \%)$ & $176(10.1 \%)$ & \\
\hline \multicolumn{5}{|l|}{ Cause of Death } \\
\hline Infectious & $20(1.3 \%)$ & $3(1.2 \%)$ & $23(1.3 \%)$ & $1.13(0.33,3.92)$ \\
\hline Neoplastic & $42(2.9 \%)$ & $5(2.1 \%)$ & $47(2.7 \%)$ & $0.99(0.38,2.57)$ \\
\hline Neurologic & $4(0.3 \%)$ & $1(0.4 \%)$ & $5(0.3 \%)$ & $1.55(0.17,14.0)$ \\
\hline Cardiovascular & $81(5.4 \%)$ & $21(8.7 \%)$ & $102(5.8 \%)$ & $1.94(1.15,3.30)$ \\
\hline Stroke & $11(0.7 \%)$ & $7(2.9 \%)$ & $18(1.0 \%)$ & $4.77(1.80,12.7)$ \\
\hline Respiratory & $9(0.6 \%)$ & $8(3.3 \%)$ & $17(1.0 \%)$ & $7.02(2.64,18.7)$ \\
\hline Gastrointestinal & $4(0.4 \%)$ & $3(1.2 \%)$ & $7(0.4 \%)$ & $5.55(1.21,25.5)$ \\
\hline Renal Failure & $10(0.7 \%)$ & $1(0.4 \%)$ & $11(0.6 \%)$ & $0.74(0.10,5.85)$ \\
\hline Non-Specific & $15(1.0 \%)$ & $8(3.3 \%)$ & $23(1.3 \%)$ & $4.27(1.74,10.5)$ \\
\hline
\end{tabular}

Abbreviations: $\mathrm{CI}=$ confidence interval; ICD-9CM = International Classification of Diseases, Ninth Revision, Clinical Modification; OR=odds ratio 
multinomial logistic regression models adjusted for age and gender, depressive symptoms were significantly more likely in those who later died from cardiovascular diseases, stroke, respiratory diseases, or non-specific causes. However, depressive symptoms were not associated with deaths due to infection or neoplasm. Few deaths were due to neurologic conditions, renal failure or gastrointestinal diseases; these results should thus be interpreted with caution.

\section{Discussion}

As we have previously reported, depressive symptoms predict all-cause mortality (4). Here, we have examined the specific causes of death associated with depressive symptoms. Depressive symptoms seem to be more strongly associated with cardiovascular and cerebrovascular deaths than deaths due to neoplasms or infections. Deaths due to dementia, renal failure or gastro-intestinal diseases were infrequent and thus their association with depressive symptoms could not be reliably determined.

There are both strengths and limitations to our approach. First, a substantial number of individuals declined consent for death certificate data, and these data were differentially missing. Second, the exact cause of death may be difficult to ascertain in older adults, particularly among those with multimorbidity where there may be multiple factors leading to death. Ascribing a particular cause of death may be difficult. Moreover, some causes of death may simply be the last precipitating event in a long illness. For instance, pneumonia may be coded as the ultimate cause of death in those with dementia. Third, these data are from the 1990s and the causes of death may have changed in the intervening years. However, it is less likely that the association between depressive symptoms and the cause of death has changed dramatically in these years. Fourth, there may not be adequate power to detect differences for some causes of death. Finally, we do not have a measure of major depression, which may be more strongly linked to death than depressive symptoms (5). Strengths include a representative population-based sampling frame, the collection of standardized valid measures by trained interviewers, and complete follow-up for death.

Our results are consistent with studies in younger adults that show an increased risk of accidental and cardiac deaths in those with depression (6). As well, other studies show an increased risk of death from stroke (7), obstructive lung disease (8) and ischemic heart disease
(9) among those with depression. However, others have found that depressive symptoms predict death from cancer (10), which our study did not. Most of these studies were over shorter time frames than we have considered.

These results may be important in planning health services and in clinical care. If depressive symptoms are modifiable risk factors for mortality, then interventions at modifying depressive symptoms at a population or an individual level may have larger effects on cardiovascular, cerebrovascular and non-specific death than cancer or infectious death. The results also highlight the link between cardiovascular risk factors, cardiac disease, and mortality. While interventions aimed at reducing depressive symptoms have had disappointing results, further inquiry into these associations remains important.

\section{Disclosures: None of the authors have any conflicts of interest to declare.}

Funding Acknowledgement: The Manitoba Study of Health and Aging (MSHA) was funded primarily by Manitoba Health (1990-93). Additional funding was provided through the Canadian Study of Health and Aging (CSHA) by the Seniors Independence Research Program of the National Health Research and Development Program of Health Canada (Project No. 6606-3954-MC(S)). The follow-up of the MSHA (MSHA-2) was funded primarily by Manitoba Health's Healthy Communities Development fund. The analysis reported here was supported by a New Emerging Team (Net) grant funded by CIHR's Institute of Aging and the Rural and Northern Health Research Initiative (HAS-63179). The results and conclusions are those of the authors and no official endorsement by Manitoba Health or other funding agencies is intended or should be inferred.

Ethical standard: This study was approved by the Research Ethics Board of the Faculty of Medicine of the University of Manitoba, and adhered to the Declaration of Helskinki.

\section{References}

1. Cuijpers P, Vogelzangs N, Twisk J, et al: Comprehensive Meta-Analysis of Excess Mortality in Depression in the General Community Versus Patients With Specific Illnesses. Am J Psychiatry 2014; 10

2. Canadian study of health and aging: study methods and prevalence of dementia. CMAJ 1994; 150 (6):899-913

3. Radloff L: The CES-D Scale: A self-report depression scale for research in the general population. Applied Psychological Measurement 1977; 1:385-401

4. St John PD, Montgomery PR: Do depressive symptoms predict mortality in older people? Aging Ment Health 2009; 13 (5):674-681

5. Cuijpers P, Vogelzangs N, Twisk J, et al: Differential mortality rates in major and subthreshold depression: meta-analysis of studies that measured both. $\mathrm{Br}$ J Psychiatry 2013; 202 (1):22-27

6. Wyman L, Crum RM, Celentano D: Depressed mood and cause-specific mortality: a 40-year general community assessment. Ann Epidemiol 2012; 22 (9):638-643

7. Pan A, Sun Q, Okereke OI, et al: Depression and risk of stroke morbidity and mortality: a meta-analysis and systematic review. JAMA 2011; 306 (11):12411249

8. de Voogd JN, Wempe JB, Koeter GH, et al: Depressive symptoms as predictors of mortality in patients with COPD. Chest 2009; 135 (3):619-625

9. Leung YW, Flora DB, Gravely S, et al: The impact of premorbid and postmorbid depression onset on mortality and cardiac morbidity among patients with coronary heart disease: meta-analysis. Psychosom Med 2012; 74 (8):786-801

10. Pinquart M, Duberstein PR: Depression and cancer mortality: a metaanalysis. Psychol Med 2010; 40 (11):1797-1810 